
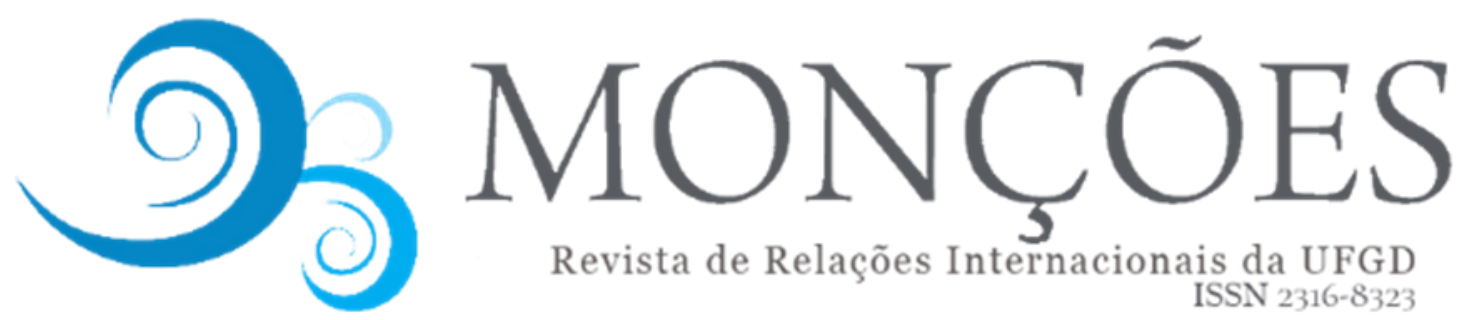

Revista de Relações Internacionais da UFGD

ISSN 2316-8323

\title{
OS OBJETIVOS DO DESENVOLVIMENTO SUSTENTÁVEL E A SAÚDE MENTAL: DISCUSSÕES À LUZ DO CONCEITO DE DESENVOLVIMENTO SUSTENTÁVEL EM CONTEXTOS AMAZÔNICOS
}

\author{
MATHEUS DOS SANTOS DA SILVEIRA \\ Núcleo de Teoria e Pesquisa do Comportamento (NTPC), Universidade \\ Federal do Pará, Belém (PA), Brasil. Bolsista de Pós-Graduação pela CAPES. \\ ORCID: https://orcid.org/0000-0003-4724-4443 \\ silveiramath49@gmail.com
}

NATÁLIA CARVALHO VIANA DE SOUSA

Núcleo de Teoria e Pesquisa do Comportamento (NTPC), Universidade Federal do Pará, Belém (PA), Brasil. Bolsista de Pós-Graduação pelo CNPQ.

ORCID: http://orcid.org/0000-0001-8636-2675

natalcvs@gmail.com

\begin{abstract}
Resumo: Desde o final do século passado o mundo vive a necessidade de reconstruir suas definições acerca do desenvolvimento. Todavia, somente a definição de sustentabilidade não se fez suficiente às reformulações necessárias no desenvolvimento das nações. Com isso foram criadas medidas gerais que funcionam como parâmetros ao novo modelo de desenvolvimento. Denominadas de Objetivos do Desenvolvimento Sustentável, compostos por 17 objetivos, as ODS servem como norteadores para tomada de decisão em inúmeros aspectos que interferem na noção de sustentabilidade, entre elas a promoção da saúde. Sabe-se que o conceito de saúde é amplo e abarca inúmeros fatores; a saúde mental é um deles e encontra-se em constante questionamento por sua invisibilidade em pautas sustentáveis e documentos internacionais, contrariando dados alarmantes de adoecimento em todo mundo. Frente ao questionamento sobre a importância de discussões e reformulações acerca do tema, o presente artigo buscou discutir o conceito de saúde mental nos Objetivos do Desenvolvimento Sustentável, utilizando a Amazônia enquanto recorte contextual, tendo em vista a diversidade da região.
\end{abstract}

Palavras-chave: Objetivos do Desenvolvimento Sustentável; Amazônia; Saúde Mental.

\section{THE SUSTAINABLE DEVELOPMENT GOALS AND MENTAL HEALTH: DISCUSSIONS IN THE LIGHT OF THE SUSTAINABLE DEVELOPMENT CONCEPT IN AMAZON CONTEXTS.}

\begin{abstract}
Since the end of the last century, the world has been in need of reconstructing its definitions of development. However, only the definition of sustainability was not enough for the necessary development reformulations of nations, with that, general measures were created, acting as parameters for a new development model, called Sustainable Development Goals, comprised of 17 objectives, the SDGs serve as guidelines for decision-making in numerous aspects that interferers in the notion of sustainability, among them health promotion. It is known that the concept of health is broad and encompasses countless factors; mental health is one of them and is in constant questioning, due to its invisibility in sustainable guidelines and international documents, contradicting worldwide alarming data of illness. Faced with the questioning of the importance of mental health in sustainability, this article sought to discuss the concept of mental health in the Sustainable Development Goals, using the Amazon as a contextual focus, in view of the region's diversity.
\end{abstract}

Keywords: Sustainable Development Objectives; Amazon; Mental Health. 


\section{LOS OBJETIVOS DE DESARROLLO SOSTENIBLE Y SALUD MENTAL: DEBATES A LA LUZ DEL CONCEPTO DE DESARROLLO SOSTENIBLE EN CONTEXTOS AMAZÓNICOS.}

Resumen: Desde fines del siglo pasado, el mundo ha necesitado reconstruir sus definiciones de desarrollo. Sin embargo, solo la definición de sustentabilidad no fue suficiente para las reformulaciones de naciones, por lo que se crearon medidas generales, actuando como parámetros para un nuevo modelo de desarrollo, denominado Objetivos de Desarrollo Sostenible. Compuesto por 17 objetivos, los ODS sirven como pautas para la toma de decisiones, incluida la promoción de la salud. Sabemos que el concepto de salud es amplio y engloba innumerables factores; La salud mental es una de ellas y está en constante cuestionamiento, debido a su invisibilidad en las guías y documentos internacionales, contradiciendo datos alarmantes de enfermedad en todas partes. Ante el cuestionamiento de la importancia de la salud mental en la sustentabilidad, este artículo buscó discutir el concepto de salud mental en los objetivos del desarrollo sustentable, utilizando la Amazonía como enfoque contextual, ante la diversidad de la región.

Palabras clave: Objetivos de Desarrollo Sostenible; Amazonia; Salud mental.

\section{Introdução}

Considerada uma das principais bases constituintes do desenvolvimento humano pleno, a saúde vem cada vez mais sendo compreendida sob uma perspectiva pluridimensional, ou seja, de que vários aspectos a compõe, se afastando do entendimento clássico de saúde enquanto algo unicamente fisiológico. Questões relacionadas à saúde, nesse sentido, são cada vez incorporadas com maior frequência nas discussões políticas, tanto em nível regional quanto internacional, considerando o que autores como Youde (2016) afirmam ao pontuar que a saúde, atualmente, é vista como um assunto de high politics, ou seja, de alta relevância na arena política.

A maior expressão de articulação internacional para garantia de desenvolvimento humano pleno da sociedade internacional talvez seja a criação dos Objetivos do Desenvolvimento Sustentável (ODS), um guia composto por 17 Objetivos e 169 metas para sustentabilidade no mundo, estabelecido pela Agenda 2030, nos quais a saúde possui uma significativa relevância, destacada por meio da ODS de número 03 ("assegurar uma vida saudável e promover o bem-estar para todas e todos, em todas as idades").

Dentro da ODS em questão, diversos indicadores e metas são propostos, com o intuito de operacionalizar o alcance de tal objetivo por parte dos Estados-membros da Organização das Nações Unidas (ONU). E, mesmo com a natureza global da 
aplicação do objetivo, esse deve dialogar com políticas e ações nos âmbitos regionais e locais, a fim de promover a atuação dos governantes e gestores locais enquanto protagonistas em torno da Agenda 2030 (PROGRAMA DAS NAÇÕES UNIDAS PARA O DESENVOLVIMENTO, 2020).

Importante pontuar que, embora ações voltadas para saúde estejam concentradas na ODS de número 03, elas não estão restritas somente a esse objetivo, sendo interrelacionadas a várias problemáticas abordadas por outras ODS, tais como: “(...) erradicação da pobreza (Objetivo 1), segurança alimentar (Objetivo 2), saneamento básico (Objetivo 6) e desigualdade entre os países (Objetivo 10)", por exemplo (DJONú et al, 2018, p. 2). Os autores também apontam que, não se deve desconsiderar que os Objetivos do Desenvolvimento Sustentável são resultados dos territórios e das sociedades, ou seja, devem ser analisados a partir das realidades locais dos Estados. Estes por sua vez, devem ser considerados em suas singularidades com objetivo de integração a um desenvolvimento no âmbito global.

Dentro da ODS de número 03, uma das metas é reduzir em um terço a mortalidade prematura por doenças não transmissíveis via prevenção e tratamento, e promover a saúde mental e o bem-estar (ORGANIZAÇÃO DAS NAÇÕES UNIDAS, 2019). Assim como a relação entre a ODS 03 e os demais Objetivos, no intuito de compreender a saúde como um constructo multidimensional, a saúde mental também surge como demanda global de medidas para o desenvolvimento das nações, e deve ser analisada em uma perspectiva dialética, a partir de aspectos individuais e contextuais (GIANNOU, 2017; SILVEIRA, 2018).

Considerando essa problemática, o presente estudo objetiva discutir, no contexto amazônico, as relações entre os Objetivos do Desenvolvimento Sustentável e o enquadramento da saúde mental enquanto aspecto de desenvolvimento sustentável na região. Embora outros estudos já tenham se detido a investigar a importância da saúde mental nas ODS (VOTRUBA; THORNICROFT, 2016; CRATSLEY; MACKEY, 2018), não foram encontradas investigações que se detivessem às realidades brasileiras, logo, nenhuma relacionada à região amazônica.

Para alcançar tal objetivo, o artigo se encontra disposto na seguinte estrutura: o segundo tópico foi construído a partir da discussão do conceito de desenvolvimento sustentável, suas mudanças ao longo do tempo; o terceiro tópico aborda a construção histórica dos Objetivos do Desenvolvimento Sustentável, com ênfase no objetivo 03, 
espaço no qual a saúde mental se faz presente; em seguida, o ideário em saúde mental será discutido sob a perspectiva de produção da (e para a) realidade amazônica. O tópico seguinte analisará como uma compreensão amazônica acerca de desenvolvimento sustentável apresenta ferramentas para a discussão da relação entre saúde mental e as ODS; por fim, as considerações finais irão apontar caminhos teórico-metodológicos a partir do apresentado.

\section{Desenvolvimento Sustentável: da noção de desenvolvimento às demandas sociais sobre meio ambiente}

O construto desenvolvimento é pluridimensional, podendo ser vislumbrado em contextos individuais, sociais e econômicos. Por ser plural, suas nuances carregam implicações e divergências relacionadas a desempenho, crescimento e satisfação. Nessa perspectiva, Furtado (1972) alega que, um desenvolvimento stricto sensu não é eficiente economicamente se acarreta somente interesses relacionados ao lucro e ao crescimento financeiro. Logo, o mais sensato e contemplativo seria afirmar que, desenvolvimento econômico constitui-se em uma utilização racional de recursos, com benefícios sociopolíticos e incrementos capitais. Contudo, este conceito não é suficiente.

No que diz respeito a necessidades elementares de populações humanas e o desenvolvimento, Furtado (1982) afirma ainda que o conceito perde a nitidez na análise dos contextos culturais caso não contemple o essencial ao desenvolvimento de uma população (habitação, educação, saúde, etc.), tornando-se apenas referência a um sistema de valores, algo "fora de contexto". Esta afirmativa garante que não existe desenvolvimento sem que haja o entendimento de particularidades sociais, ambientais e culturais de diferentes contextos humanos.

Sendo assim, o presente estudo advoga uma perspectiva de enfoque do desenvolvimento humano à luz das necessidades humanas fundamentais, ou seja, aspectos, tangíveis e/ou intangíveis, relativos à vida individual e social que permite, a partir de sua satisfação, a construção de processos de desenvolvimento humano. Logo, o construto desenvolvimento pode ser entendido não apenas como algo vinculado às políticas internacionais e nacionais, mas também a indicadores próprios da vida das pessoas. 
Max-Neef, Elizalde e Hopenhayn (2010) atestam tal afirmação ao pontuarem como o desenvolvimento deveria ser direcionado às pessoas e não aos objetos, em uma clara crítica ao modo como as políticas desenvolvimentistas pautavam seus discursos em uma tentativa de contribuição à vida das pessoas, quando, de fato, ações eram vistas apenas em níveis macroscópicos, e não na realidade diária dos grupos sociais. Era necessário, segundo os autores, proposições que migrassem de justificativas e motivações econométricas, de ações que fossem para além de uma lógica estadocêntrica. Mudanças estas que, segundo eles, iriam compor um "desenvolvimento à escala humana".

A partir dessas proposições, os autores expuseram que a maioria dos estudos sobre desenvolvimento humano hesita em postular que as necessidades humanas são infinitas, que mudam constantemente de diferentes ambientes, culturas e períodos históricos. As necessidades humanas fundamentais são finitas, em pequeno número, sujeitas a classificação, estáveis em todas as culturas e períodos históricos (MAX-NEEF; ELIZALDE; HOPENHAYN, 2010). O que realmente muda é como essas necessidades são atendidas, ou seja, a maneira ou os meios utilizados para satisfazer as necessidades. Daí uma importante distinção conceitual no estudo das necessidades humanas: necessidades e satisfações.

Satisfazemos (ou não) as necessidades fundamentais da existência a partir das adotadas pelas estruturas econômicas, sociais e políticas atuais; portanto, ao analisar as necessidades fundamentais de um grupo social específico, é necessário investigar se essas necessidades estão sendo atendidas em uma base estável de tempo, como elas estão sendo atendidas (de que forma e por quais meios) e, se não, porque deixam de ser atendidas (MAX-NEEF; ELIZALDE; HOPENHAYN, 2010; PELENC; DUBOIS, 2020).

De modo geral, as necessidades humanas fundamentais podem ser compreendidas enquanto um conjunto de aspectos tangíveis e intangíveis, simultâneos, complementares e compensatórios, relativos à vida individual e social e que permitem os processos de desenvolvimento (MAX-NEEF; ELIZALDE; HOPENHAYN, 2010; MORENO; SILVA; SOUZA, 2017). Simultâneos, pois demandamos em nossas vidas a presença de mais de uma necessidade ao mesmo tempo; complementares, pois um pleno desenvolvimento humano se dá a partir da complementaridade de distintas necessidades satisfeitas e compensatórias, pois as 
necessidades fundamentais operam em um equilíbrio alcançado entre aspectos desejáveis, mas incompatíveis.

A partir desta fundamentação, Max-Neef, Elizalde e Hopenhayn (2010) criaram o que foi denominada Matriz de Necessidades e Satisfatores, que a partir das contribuições de autores como Abraham Maslow e Dag Hammarskjöld, classificou as necessidades humanas fundamentais e possíveis satisfatores para cada uma. Portanto, as necessidades poderiam ser divididas em dois domínios: a axiológica, composta pelas categorias de subsistência, proteção, afeto, entendimento, participação, ócio, criação, identidade e liberdade; e a existencial, composta pelas categorias de ser, ter, fazer e interagir ${ }^{1}$. Embora um dos pontos levantados seja 0 fato de que todas as necessidades são importantes (logo, fundamentais), a categoria de subsistência acaba assumindo uma posição hierárquica em relação às demais, isso se dá ao fato de que, essa categoria está relacionada à necessidade humana de se manter vivo.

Entretanto, a discussão contemporânea acerca da satisfação das necessidades humanas fundamentais está relacionada ao modo em que as medidas construídas para tal objetivo contribuem para a degradação do planeta, em uma perspectiva socioambiental (VITA; HERTWICH; STADLER; WOOD, 2019). Preocupações em torno do "imperativo da satisfação individual" frente à conservação de recursos para futuras gerações se difundiram, em especial a partir da segunda metade do século $X X$, gerando, dessa forma, a necessidade por respostas articuladas internacionalmente (GUILLEN-ROJO; GUARDIOLA; GARCIA-QUERO, 2017; VITA; HERTWICH; STADLER; WOOD, 2019)

Fruto dessa inquietação, e consequente conscientização social global ocorrida no final do século XX, o conceito de Desenvolvimento Sustentável surgiu contrapondo os processos de desenvolvimento advindos da revolução industrial. Van Bellen (2004) alega que a crise ambiental e a influência da sociedade, foram essenciais para a criação do termo, que surge a partir da década de 1990, na redefinição de um modelo antidesenvolvimentista convencional.

Os movimentos de pós-guerra, e a segunda metade do século $X X$ foram extremamente significativos para novos rumos e definições do que seria esperado

\footnotetext{
${ }^{1}$ Para uma compreensão mais detalhadas de tais categorias, ver Max-Neef, Elizalde e Hopenhayn (2010).
} 
enquanto desenvolvimento. A preocupação sobre o uso saudável e sustentável do planeta, bem como de seus recursos, já era uma realidade crescente, e no ano de 1972 a ONU convoca a Conferência das Nações Unidas sobre o ambiente Humano, em Estocolmo, na Suécia. Essa conferência representou os esforços iniciais de práticas comuns às nações para preservação ambiental, com um Manifesto Ambiental que continha 19 princípios para o novo século (ORGANIZAÇÃO DAS NAÇÕES UNIDAS, 2019).

\section{Das Metas do Milênio aos Objetivos do Desenvolvimento Sustentável: atravessamentos da perspectiva global em saúde}

Com a criação do Relatório Nosso Futuro Comum, houveram inúmeras medidas para se pensar o que seria esse "novo modelo" de desenvolvimento para o mundo. No inteire da discussão, surge na Conferência Rio 92, a Agenda 21, a primeira carta de intenções para promover, em escala planetária, um novo padrão de desenvolvimento para o século XXI. Ao final deste documento abriu-se o debate para necessidade de estabelecer metas comuns à todas as Nações com relação ao Desenvolvimento Sustentável para todos (ORGANIZAÇÃO DAS NAÇÕES UNIDAS, 2019).

Após as decisões tomadas do final da década de 1990, surge nos anos 2000, a discussão sobre os Objetivos de Desenvolvimento do Milênio (ODM), ou simplesmente, Metas do Milênio. As metas foram oito medidas adotadas pelos Estados-Membros da ONU e que impulsionariam esses países no enfrentamento dos principais desafios do início do século XXI.

Os objetivos do Desenvolvimento do Milênio seriam então: erradicar a extrema pobreza e a fome; atingir o ensino básico universal; promover a igualdade de gênero e a autonomia das mulheres; reduzir a mortalidade infantil; melhorar a saúde materna; combater o HIV/AIDS, a malária e outras doenças; garantir a sustentabilidade ambiental; estabelecer uma parceria mundial para o desenvolvimento. Esses objetivos deveriam ser cumpridos até 0 ano de 2015 (COMPÊNDIO PARA SUSTENTABILIDADE, 2007).

Após a Rio+20, Conferência que ocorreu no ano de 2012, no Rio de Janeiro, houve a necessidade de repensar as metas propostas e reformular a Agenda de 
Desenvolvimento Global. Então, no ano de 2015, foi adotada e implementada, em uma Assembleia da ONU, uma nova Agenda de Desenvolvimento global, chamada de "Transformando Nosso Mundo". A Agenda de 2030, entrou em vigor em 2016, com um guia composto de 17 Objetivos do Desenvolvimento Sustentável (ODS), e 169 metas para o desenvolvimento dos Estados-Membros até 2030.

De acordo com o Programa das Nações Unidas Para o Desenvolvimento (PNUD), os 17 ODS foram formulados com base nos oito Objetivos do Milênio (ODM), de forma a complementar o trabalho iniciado por eles e responder aos novos desafios. Com características integradas e indivisíveis, os ODS mesclam as três dimensões do Desenvolvimento Sustentável: os fatores econômicos, sociais e ambientais (PROGRAMA DAS NAÇÕES UNIDAS PARA O DESENVOLVIMENTO, 2019)

Ademais, o princípio "Do global para o local" foi estabelecido enquanto base dos ODS, e de acordo com o PNUD, embora exista uma natureza global na aplicação dos objetivos, eles dialogam com as políticas e ações nos âmbitos regionais e locais, com o objetivo de promover a atuação dos governantes e gestores locais enquanto protagonistas em torno da Agenda 2030 (PROGRAMA DAS NAÇÕES UNIDAS PARA O DESENVOLVIMENTO, 2020).

Os 17 objetivos são interconectados e indissociáveis, ou seja, o sucesso de um ODS envolve o combate a temas que estão associados a outros objetivos. Todavia, considerando o foco do presente artigo, é necessário realizar uma descrição com maior detalhamento da ODS de número 03, referente à saúde e bem-estar. A Agenda 2030 prevê que, o desenvolvimento das ações propostas ${ }^{2}$ contribua em assegurar

\footnotetext{
${ }^{2}$ a) até 2030 , reduzir a taxa de mortalidade materna global para menos de 70 mortes por 100.000 nascidos vivos;

b) até 2030, acabar com as mortes evitáveis de recém-nascidos e crianças menores de 5 anos, com todos os países objetivando reduzir a mortalidade neonatal para pelo menos 12 por 1.000 nascidos vivos e a mortalidade de crianças menores de 5 anos para pelo menos 25 por 1.000 nascidos vivos; c) até 2030, acabar com as epidemias de AIDS, tuberculose, malária e doenças tropicais negligenciadas, e combater a hepatite, doenças transmitidas pela água, e outras doenças transmissíveis;

d) até 2030, reduzir em um terço a mortalidade prematura por doenças não transmissíveis via prevenção e tratamento, e promover a saúde mental e o bem-estar;

e) reforçar a prevenção e o tratamento do abuso de substâncias, incluindo o abuso de drogas entorpecentes e uso nocivo do álcool;

f) até 2020 , reduzir pela metade as mortes e os ferimentos globais por acidentes em estradas;

g) até 2030, assegurar o acesso universal aos serviços de saúde sexual e reprodutiva, incluindo o planejamento familiar, informação e educação, bem como a integração da saúde reprodutiva em estratégias e programas nacionais;

h) atingir a cobertura universal de saúde, incluindo a proteção do risco financeiro, o acesso a serviços de saúde essenciais de qualidade e o acesso a medicamentos e vacinas essenciais seguros, eficazes, de qualidade e a preços acessíveis para todos;
} 
uma vida saudável e promover o bem-estar para todas e todos, em todas as idades (ORGANIZAÇÃO DAS NAÇÕES UNIDAS, 2019):

Um dos indicadores de alcance do objetivo 03 é visto a partir da promoção da saúde mental e do bem-estar das populações. A inserção deste indicador, como apontado por estudos da área, foi resultado de esforços coletivos, interdisciplinares e internacionais, advogando pela importância da inclusão da saúde mental entre os ODS, tendo em vista sua articulação com aspectos de âmbitos econômicos, sociais e culturais (THORNICROFT; PATEL, 2014; GUREJE; TRORNICROFT, 2015; LUND et al., 2018; SAXENA et al., 2020).

Contudo, tal advocacia data de muito antes da proposição dos Objetivos do Desenvolvimento Sustentável. Como apresentado por Patel, Saxena, Frankish e Boyce (2016), a revista The Lancet, uma das mais renomadas revistas científicas no mundo, publicou em 2007 um conjunto de artigos sobre saúde mental global, concluindo a edição com uma proposição de ação que objetivava desenvolver os serviços de saúde mental a partir dos princípios de cuidado baseados em evidência e da dignidade humana. A partir da publicação, pesquisadores de diversos centros ao redor do mundo, intensificaram seus posicionamentos frente à urgência de que as políticas sistemáticas em saúde levassem em consideração o cuidado à saúde mental, em especial de grupos em situação de vulnerabilidade social (THORNICROFT; PATEL, 2014; VOTRUBA; PRINCE; THORNICROFT, 2014; LUND et al., 2018; MILLS, 2018).

De fato, como pontuam Mclnnes e Lee (2012), doenças infecciosas geram maiores repercussões, e consequentemente, maior financiamento para estudos que

\footnotetext{
i) até 2030, reduzir substancialmente o número de mortes e doenças por produtos químicos perigosos, contaminação e poluição do ar e água do solo;

j) fortalecer a implementação da Convenção-Quadro para o Controle do Tabaco em todos os países, conforme apropriado;

k) apoiar a pesquisa e o desenvolvimento de vacinas e medicamentos para as doenças transmissíveis e não transmissíveis, que afetam principalmente os países em desenvolvimento;

I) proporcionar o acesso a medicamentos e vacinas essenciais a preços acessíveis, de acordo com a Declaração de Doha, que afirma o direito dos países em desenvolvimento de utilizarem plenamente as disposições do acordo TRIPS sobre flexibilidades para proteger a saúde pública e, em particular, proporcionar o acesso a medicamentos para todos;

m) além de aumentar substancialmente o financiamento da saúde e o recrutamento, desenvolvimento e formação, e retenção do pessoal de saúde nos países em desenvolvimento, especialmente nos países menos desenvolvidos e nos pequenos Estados insulares em desenvolvimento;

n) reforçar a capacidade de todos os países, particularmente os países em desenvolvimento, para o alerta precoce, redução de riscos e gerenciamento de riscos nacionais e globais de saúde (ORGANIZAÇÃO DAS NAÇÕES UNIDAS, 2019).
} 

outras áreas, a exemplo da saúde mental. Mais recentemente, Cooper (2016) atesta que, durante ao menos a última década, um forte movimento internacional foi responsável por "apresentar" o campo da saúde mental global para instituições de financiamento e pesquisa, principalmente no que tange aos estudos de mecanismos de redução de disparidades de saúde mental de populações correlacionadas com outras variáveis, como renda, território, faixa etária, etc.

Enquanto campo de estudos, autores da área, fortemente influenciados por movimentos como o da psiquiatria transcultural e da antropologia e sociologia da saúde, atualmente criticam determinismos biológicos que possam influenciar o desenvolvimento de ações de subtemas da área, como saúde mental em países em desenvolvimento, por exemplo (COOPER, 2016). Embora não se deva considerar o movimento enquanto homogêneo, é reconhecido que este vem questionando tradições cartesianas que há tempos atravessavam a saúde global, tais como biológico-social; global-local; conhecimento biomédico-conhecimento tradicional, dentre outros.

Tal movimento repercutiu na disseminação de investigações acerca da saúde mental global em uma perspectiva sociocultural. Embora a ONU não tenha inserido a agenda em suas Metas do Milênio, a Organização Mundial de Saúde (OMS), agência especializada da ONU em tais assuntos, já vinha manifestando a relevância da construção de uma agenda de políticas interdisciplinares e internacionais em matéria de saúde mental.

Um dos primeiros documentos advogando tal mudança são os Princípios das Nações Unidas para a Proteção de Pessoas com Enfermidades Mentais e Melhoria da Atenção à Saúde Mental. Apresentado em 1991 pela ONU, o documento é pautado em princípios como o da não discriminação, representando um marco nos estudos de saúde mental global (ORGANIZAÇÃO MUNDIAL DA SAÚDE, 2003; PATEL; COHEN; MINAS; PRINCE, 2013).

Os Princípios de 1991 se associam em um documento criado para servir de base à proposição, criação e acompanhamento de legislações nacionais em matéria de saúde mental (ORGANIZAÇÃO MUNDIAL DA SAÚDE, 2005), e abordam questões relacionadas às liberdades fundamentais e os direitos básicos de pessoas com transtornos mentais, além de pontos referentes à melhoria do acesso aos serviços de saúde mental nacional e internacionalmente. (BERTOLOTE, 1995). Entretanto, 
algumas críticas são feitas ao documento, como por exemplo, a criação de uma burocracia que, em determinadas situações, não deve existir, visando um tratamento mais próximo do adequado às pessoas que possuem algum sofrimento psíquico (ORGANIZAÇÃO MUNDIAL DA SAÚDE, 2005).

O documento Investing in Mental Health, produzido pela OMS em 2003, por sua vez, aponta noções introdutórias acerca do tema, como o conceito de saúde mental adotado pela organização, a magnitude e os limites das desordens mentais, ações realizadas para a promoção da saúde mental e qualidade de vida, prevenção e controle de desordens mentais, além da participação da OMS na melhoria da qualidade de vida de pessoas com sofrimento mental. É um dos documentos basilares para a construção desse projeto, considerando que foi um dos primeiros a apontar com ênfase os obstáculos para o acesso pleno de diversos segmentos dos indivíduos a serviços de saúde.

Em 2005, a OMS publicou o Livro de Recursos da OMS sobre Saúde Mental, Direitos Humanos e Legislação, no qual, dentre outros aspectos, como os Estadosmembro da OMS deveriam pautar suas legislações para garantir o mais completo acesso às informações e serviços de saúde mental para a sua população, apresentando os conteúdos necessários às legislações, e como essas ferramentas deveriam proceder para estarem em conformidade com os pressupostos da organização e os diversos processos de implementação em saúde mental (SILVEIRA, 2018).

Logo, é possível constatar a relevância adquirida (em especial no século XXI) da agenda em saúde mental no campo global, ou como afirmam Votruba, Eaton, Prince e Thornicroft (2014), a inserção da pauta enquanto um problema de desenvolvimento global. A apresentação de um objetivo específico sobre saúde, e um indicador referente à saúde mental repercute, nesse sentido, três pontos: em primeiro lugar, a ONU, enquanto maior organização internacional existente atualmente (em termos de representatividade), realizou severas mudanças após a análise dos avanços e das falhas das Metas do Milênio, considerando novos desafios ao desenvolvimento internacional (IZUTSU et al., 2015); tais mudanças, no entanto, não foram alcançadas sem a consistente advocacia de outras partes importantes da agenda internacional (Organizações da Sociedade Civil, redes internacionais de colaboração em Ciência \& Tecnologia, dentre outros); por fim, e talvez o mais 
importante ponto, o ideário de desenvolvimento sustentável foi posto à sistematização de fato, considerando os significados que tal constructo carrega.

Autores como Holden, Linneurd e Banister (2017) apresentam uma visão crítica em relação ao desenvolvimento sustentável: segundo eles, a aspiração em alcançar todas as possibilidades sobre "(...) o que é bom e desejável para a sociedade, os objetivos acabaram como vagos, fracos ou sem sentido"3 (p. 214). Isso se dá, pois, uma agenda à luz do ideário de desenvolvimento sustentável deve estar baseada na satisfação de necessidades humanas, na garantia de equidade social e no respeito aos limites ambientais, e, por motivações político-econômicas, isso não é visto na prática.

O distanciamento entre a constituição do discurso e sua real efetivação é vista em especial quando as análises se voltam para grupos em vulnerabilidade. A região amazônica não é exceção, considerando forças externas podem ser internalizadas pelas comunidades locais, moldando, dessa forma, as relações de poder existentes (TALLMAN, 2019). O desenvolvimento de espaços de vulnerabilidade se dá, neste sentido, em uma relação na qual fatores como incerteza e imprevisibilidade, e estigmas sociais construídos atravessam as políticas públicas vinculadas à região, sobre o que é visto como relevante e o que não é (WUTICH; BREWIS, 2014).

De fato, ao analisarmos como os Objetivos do Desenvolvimento Sustentável são atravessados nas realidades amazônicas, é importante compreender que, embora as políticas públicas advindas tenham um caráter universal, a realidade apresenta que estas esbarram em problemáticas locais e regionais. Logo, é importante olhar para a região a partir de ferramentas teórico-metodológicas que considerem a bidirecionalidade entre as dinâmicas locais e globais (SCHWEICKARDT et al, 2019).

Tal necessidade é ainda mais importante quando a análise se foca em um tema ainda não discutido como se deveria, a exemplo da saúde mental. Mesmo que os discursos sobre um desenvolvimento dinâmico, multicêntrico e sistêmico apareça com intensidade cada vez maior nas produções sobre o tema, a temática saúde mental simplesmente não é inserida, ou, quando é, isso ocorre de uma forma extremamente

\footnotetext{
3 "By attempting to cover all that is good and desirable in society, these targets have ended up as vague, weak, or meaningless" (2017, p. 213)
} 

APLICADA, 2019; VIEIRA, 2019; TALLMAN, 2019)

\section{Saúde Mental nas Realidades Amazônicas}

Antes de abordar a questão da saúde mental propriamente dita, é importante salientar alguns pontos relacionados a construção do saber sobre a região. O ponto principal a ser questionado para reformulação do ideal de desenvolvimento para Amazônia está relacionado com a pluralidade de contextos existentes na região, enquadrando-a como "Amazônias", por diversidade de fauna, flora, estados abrangentes, populações, e principalmente as diferenças culturais existentes. Contemplar esta região em cenários e realidades múltiplas que foram construídas em muitos aspectos, a base de violência e colonizações (LOUREIRO, 2002), nos confere o entendimento de que as demandas de saúde podem ser extremamente diversas e particulares, e quando abordamos saúde mental, essas diferenças acentuam-se ainda mais.

Neste sentido, a construção do construto saúde mental, deve ser encarada como um assunto de todos: desde indivíduos até Organizações Internacionais, considerando a necessidade de uma linguagem unificada e pluridimensional para a aplicação da teoria em políticas públicas, um sentido integrador no compartilhamento dos recursos destinados às ações, além de participação integral de todos os setores da sociedade internacional para a maior efetividade (ORGANIZAÇÃO MUNDIAL DA SAÚDE, 2004).

As discussões sobre os espaços de cuidado em saúde mental em contextos amazônicos devem, necessariamente, perpassar pelo modelo de atenção construído após a chamada Reforma Psiquiátrica, que objetivou processos de desinstitucionalização das pessoas em sofrimento psíquico, bem como um cuidado mais integral da sociedade, tendo em vista uma perspectiva sociológica da saúde. Contudo, esse modelo ainda esbarra em operantes políticos que dificultam o pleno desenvolvimento das ações, tais como a desarticulação, em muitos momentos, de ações das esferas municipal, estadual e federal (BATISTA; FERREIRA; BATISTA, 2018). 
Os entraves relacionados a participação do estado são acentuados em regiões que apresentam baixa concentração demográfica, como a região amazônica, em sua maioria. Como afirmam Batista, Ferreira e Batista (2018), a dinâmica de recursos muitas das vezes não favorece o desenvolvimento de ações efetivas presentes no cerne de políticas para saúde mental. Logo, é possível perceber um descompasso entre o que se é proposto e o que é possível ser executado. Este aspecto pode ser considerado estrutural no que diz respeito a implementação de medidas de saúde em demais políticas públicas.

O estudo de Silveira e Pinheiro (2014) aponta que, a partir da realidade do território brasileiro, a região que mais carece de serviços de saúde é a região amazônica, principalmente nas cidades do interior. Tais dados reforçam o estereótipo conferido à região enquanto um lugar de falta, de ausência (SCHWEICKARDT et al, 2019). É possível apreender, com este aspecto, a ainda distante discussão sobre como as realidades locais são analisadas pelas lentes dos discursos globais, tendo em vista que, em muitas das vezes, as reivindicações atravessam a falta de dialética nas agendas internacionais.

Sabe-se que, as demandas de saúde em contextos amazônicos são permeadas, principalmente, por questões estruturais relacionadas à saneamento básico, distribuição de renda, pobreza, fome e doenças infectocontagiosas, que em muitos casos estão atreladas a aspectos educativos, a saúde da família e a atenção primária em saúde (ANDRADE, 2007; MEDEIROS et al., 2018; VIEIRA; GARNELO; HORTALE, 2016). Todavia, esses aspectos estruturais também refletem em demandas da saúde mental na região, como os altos índices de alcoolismo (MORETTI-PIRES; CORRADI-WEBSTER; MENDONÇA, 2011), depressão, transtorno de ansiedade, esquizofrenia, psicose, o uso de drogas e a violência em populações tradicionais e em etnias indígenas (BATISTA; ZANELLO, 2016).

Neste sentido, é importante destacar que a literatura aponta as limitações encontradas na operacionalização de planos e programas específicos em saúde mental, tanto a nível local, regional, quanto nacional e internacional (VÉLEZ et al., 2020). De fato, os autores pontuam que as articulações entre saúde mental e diversidade sociocultural se limitam (quanto aparecem) em ações pontuais, a exemplo da gestão de riscos e da invisibilidade do viés cultural (BATISTA; ZANELLO, 2016). Uma análise no Plano Amazônia Sustentável, programa desenvolvido pelo Governo 
Federal em 2008 com um conjunto de diretrizes para o desenvolvimento sustentável na Amazônia Brasileira, ratifica tal afirmação.

O Plano apresenta, ao discutir sobre saúde na região, onze diretrizes tidas como "(...) resposta às reivindicações da sociedade amazônica" (2008, p. 74), considerando o Plano de Qualificação da Atenção à Saúde na Amazônia Legal (Plano Saúde Amazônia), criado em 2006. Em nenhuma das onze diretrizes, ações em saúde mental são postas enquanto prioridades, direta ou indiretamente, caracterizando, neste sentido, uma noção reducionista de saúde, e para além, de desenvolvimento sustentável. Madeira (2014) reitera tal questionamento afirmando a noção institucionalizada de desenvolvimento sustentável apresentada pelo Plano, não levando em consideração, na prática, demandas da realidade das comunidades amazônicas. Logo, (...) "as populações da maior parte da Amazônia continuam a se defrontar com incontornáveis barreiras" (p. 28).

Estudos conduzidos por Tallman (2019), Wutich e Brewis (2014) e Leatherman, Hoke (2016) e Batista e Zanello (2016) apontam a vulnerabilidade dos indivíduos e os espaços de vulnerabilidade enquanto marcadores sociais de identidade e de saúde mental. Mesmo que os contextos amazônicos sejam percebidos mundialmente como espaços de grande diversidade cultural, esse aspecto não consegue ser traduzido em planos ou medidas que garantam a integralidade na saúde, o que geram discursos, medidas e políticas que de algum modo geram a invisibilidade das populações.

Neste sentido a contextualização da saúde é uma das maiores prerrogativas ao desenvolvimento sustentável na região, pois ela reflete uma adequação das diferenças em relação a um território pluridimensional (LOUREIRO, 2002). Essa contextualização nos permite ir além quando nos deparamos com o conceito de sustentabilidade, de suprir necessidades das gerações presentes sem comprometer as futuras geração. Pois permite compreender que circunstâncias sociais, econômicas e ambientais também são determinantes de saúde, e que é improvável desvincular demandas socioeconômicas e culturais de demandas de saúde, principalmente saúde mental (LUND et al., 2018).

Essa afirmação ganha ainda mais força quando comparamos os altos índices de depressão e suicídio entre indígenas a cada ano, e fatores como a demarcação de terra, crises e desamparo político, desmatamento e conflitos agrários, o preconceito 
racial, a desvalorização cultural e a forte pressão a incorporação ao mercado econômico (BATISTA e ZANELLO, 2016).

\subsection{Saúde Mental Global para Quem?}

Em um primeiro momento, é importante ressaltar a validade da compreensão da saúde em sua perspectiva global. Cueto (2015) afirma que a saúde global, enquanto campo de ações e de estudos pode ser sintetizada em duas perspectivas, sendo a primeira relativa à utilização de tecnologias modernas para o controle de doenças, sendo vinculadas ao discurso de crescimento econômico; e a segunda relacionada ao desenvolvimento de programas sociais que visem mitigar as diversas desigualdades enfrentadas por diversas populações em escala global. A criação e subsequente acompanhamento das ODS vêm representando uma ação internacional vinculada à segunda perspectiva proposta pelo autor.

No entanto, como já mencionado anteriormente, ações em saúde em uma perspectiva global devem ser problematizadas em relação a como elas se inserem nas realidades locais das sociedades. Carlos Javier Regazzoni (2007) sintetiza cinco conclusões acerca das consequências do desenvolvimento da saúde global para o século XXI: a) desde o século passado, o estado de saúde, de maneira geral, melhorou; b) essa melhora, no entanto, vem se concentrando nos países do "primeiro mundo"; c) a melhora desses padrões de saúde em escala global depende, não somente da evolução da tecnologia médica, mas também do desenvolvimento social; d) a evolução na qualidade de saúde nunca foi tão assimétrica, em termos sanitários; e) esse fenômeno reflete o fato de que a evolução de uma sociedade é muito mais complexa que aspectos estritamente médicos (p. 6).

Para além disso, o próprio conceito de saúde deve ser interrogado. Quanto a este, difundido mundialmente no preâmbulo da constituição da OMS, desde ao menos as últimas duas décadas, autores vêm exibindo críticas relativas a validade do conceito (NORDENFELT, 2007). Dentre os autores, Matcheld Huber talvez seja um dos que mais contribua para a discussão, a partir da publicação de sua tese (2014), na qual ele questiona o conceito apresentado pela OMS e propõe uma nova definição (uma conceituação dinâmica, em suas palavras). 
Segundo o autor, a principal falha do conceito é o fato da OMS apontar a palavra completo em sua definição, pois, acaba reforçando a busca por um estado impossível de ser alcançado, e logo, mensurado (NORDENFELT, 2007; HUBER, 2014). Essa busca pode ser vista no processo de medicalização da sociedade ocidental, que, embora não seja um fenômeno recente, viu no desenvolvimento das indústrias farmacêuticas seu apogeu.

A partir de conferências sobre o assunto, Huber et al. (2014) apresentaram uma definição mais dinâmica do termo saúde, este enquanto "a habilidade de adaptação e autogerenciamento" (p. 48). O "componente" mental da saúde, neste caso, estaria relacionado ao que os autores chamam por "senso de coerência", senso este responsável pela capacidade de lidar e recuperar de situações psicológicas de estresse e prevenir estresses generalizados pós-traumáticos, resultando, dessa forma, numa interação holística entre o físico e o psicológico (HUBER et al, 2014).

Questionar o conceito central de uma organização de larga escala, como a OMS, é basilar para analisar os processos de construção de práticas discursivas empreendidas pela mesma ao longo de sua história. Ainda mais relevante se dá, pois, desde a sua instituição, a OMS “(...) luta pela hegemonia no campo da saúde, tanto no aspecto político quanto na construção de normas e consensos técnicos internacionais" (MATTA, 2005, p. 374).

\subsection{O Negligenciamento do Conceito de Saúde Mental nas definições de Desenvolvimento Sustentável}

Como discutido anteriormente, a saúde é apontada como requisito primordial para o desenvolvimento sustentável. O objetivo de número três propõe medidas para assegurar uma vida saudável e promover bem-estar para todas e todos, em todas idades. De maneira geral, essas medidas contemplam em sua construção a importância de estruturar medidas que amparem diferentes gêneros e idades (ODS 3ONU, 2015).

Todavia, ao analisar os indicadores e metas, percebe-se que a saúde mental é abordada somente em um aspecto, com a redução da mortalidade prematura por doenças não transmissíveis (entre elas podemos enquadrar o suicídio), e a promoção de saúde mental e bem-estar. Ao analisarmos a complexidade da saúde mental, 
percebemos que ela é abordada nas ODS's de maneira reducionista, sendo vinculada somente a doenças não transmissíveis e a promoção de bem-estar, e que esta não dialoga com demais metas ou indicadores.

Neste aspecto, garantir uma vida saudável e promover o bem-estar para todos em todas as idades é impossível sem uma consideração da saúde mental contextual, que dialogue com a fome, pobreza, violência, consumo, e até mesmo a preservação ambiental (LUND et al., 2018). Desigualdade dentro e entre os países não podem ser totalmente abordados, a menos que reconheçamos que quase um quarto da população mundial - o número de pessoas que sofrem de uma doença mental ano experimenta discriminação sistemática na maioria das áreas da vida. Essas relações de desigualdade são agravadas quando relacionadas a questões de raça, gênero e classe social (THORNICROFT; PATEL, 2014).

A melhoria dos sistemas de saúde mental também possui um papel decisivo na construção de cidades e assentamentos humanos inclusivos, seguros, resilientes e sustentáveis. Essas ações são especialmente importantes, dada a tendência global de urbanização como um fator de risco associados à doença mental, e neste aspecto, podemos fazer um recorte à tendência de urbanização em regiões amazônicas e as dificuldades encontradas por populações tradicionais (THORNICROFT; PATEL, 2014).

Ainda sobre a necessidade de relacionar saúde mental nos mais diversos níveis de sustentabilidade, Thornicroft e Patel (2014), apontam que uma, entre tantas, relações viáveis seria a compreensão acerca do trabalho para saúde mental (ODS 8). Pessoas com doença mental têm taxas de emprego muito mais baixas que o restante da população e períodos de recessão econômica são relacionados a piores padrões de saúde na população, principalmente em homens. Em países com alta renda, cerca de um quarto das pessoas com doença mental recebe cuidados e, em países de baixa renda, menos de um em cada dez consegue entrar em contato com algum serviço em saúde mental, por exemplo.

3.3 As Implicações da(s) saúde(s) mental(is) para o Desenvolvimento Sustentável na Amazônia 
Não se pode, desse modo, desconsiderar questões socioculturais nas discussões em cuidado à saúde mental e desenvolvimento sustentável em contextos amazônicos. Mais ainda, não se pode retirar o ideário de desenvolvimento, em um sentido mais amplo, é utilizado enquanto substrato para discursos de silenciamento e reforçamento da vulnerabilidade de diversos grupos sociais. A histórica exploração por recursos naturais e humanos na região amazônica atesta o quão prejudicial tal discurso pode ser.

Um estudo realizado por Tallman (2019), por exemplo, investigou associações entre a insegurança hídrica e o estresse psicológico de 225 pessoas de quatro comunidades Awajún, na região da Amazônia peruana. Os achados apontam associações entre alto escores de insegurança hídrica e estresse percebido, sintomas depressivos e sintomas somáticos nas comunidades. Wutich e Brewis (2014), por sua vez, afirmam que fatores como incerteza e imprevisibilidade, estigmas sociais desenvolvidos, percepção de injustiça, podem estar relacionados à sofrimento psicológico. Considerando o contexto atual vivido, se torna imprescindível uma maior sistematização de tais estudos.

Relatando acerca de realidades no território brasileiro, Raquel Baster (2019) pontua como 0 alto índice de projetos de desenvolvimento (ou megaempreendimentos) desencadeia, em curto prazo, em conflitos socioterritoriais nas diversas regiões, contribuindo, em longo prazo, para o comprometimento da saúde mental das populações, e em maior âmbito, sua qualidade de vida. Tais consequências se dão, segundo Cardoso de Castro (2020, não publicado), a partir de:

(...) casos de coerção, prisões e outras violências contra mulheres no campo, assim como os efeitos diretos e indiretos dos empreendimentos no meio ambiente que afetam o cotidiano e as condições de sobrevivência das mulheres, historicamente e socialmente situadas na dimensão da produção alimentar, por exemplo. Enquanto, mais uma vez, relatam--se o aumento de riscos e casos de estupros, gravidez indesejada e o aumento de problemas relacionados à saúde mental e emocional (p.112).

Neste sentido, Batista e Zanello (2016) discutem acerca da multiplicidade do constructo vulnerabilidade, e como este acaba atravessando diversos grupos sociais, como as comunidades indígenas, por exemplo; os autores vão além, afirmando de que modo marcadores sociais de identidade também são importantes fatores de 
análise. À título de exemplificação, ao analisarmos como a sociedade amazônica, de uma forma geral, é "lida e interpretada" pelos principais atores políticos, é possível afirmarmos que existem dois tipos existentes de vulnerabilidade no que diz respeito à impactos na saúde mental: a vulnerabilidade individual e a vulnerabilidade socioespacial. Embora ambas possam fazer parte do mesmo espectro, marcadores como gênero, sexualidade, classe social, raça/etnia acabam por diferenciar as consequências de tais atos.

De acordo com Santos, Huang, Menezes e Scazufca (2016), mesmo a Amazônia enquanto região seja percebida como um lugar de grande e rica diversidade de culturas e recursos naturais, inúmeras disparidades são enfrentadas pelas populações, tais como altos índices de desemprego e pobreza, e que questões de gênero, falta de suporte social, alta exposição a eventos estressores de vida, dentre outros fatores, contribuem para a prevalência de transtornos conhecidos como depressão, ansiedade, etc.

Dito isso, é de suma importância a soma de indagações críticas que problematizem tais relações, a fim de que esse ciclo de vulnerabilidades possa ser quebrado. Como atesta Mills (2018): “(...) Suplantar um discurso estigmatizante de pobreza por um 'psicodiscurso' estigmatizante é muito problemático, especialmente quando ambos individualizam e patologizam o empobrecimento" (p. 856). Somado a isso, têm-se a dificuldade ao acesso de serviços básicos em saúde, principalmente os de saúde mental.

Analisando a realidade brasileira, Silveira e Pinheiro (2016) constataram que o acesso a profissionais de saúde, em especial médicos, atingiam os piores índices na região amazônica, com diferenças ainda mais desproporcionais em cidades do interior em relação à capitais como Belém e Manaus. Tal dado ratifica que a percepção tida da Amazônia é, em vez de uma ecologia propícia ao cuidado (MILLS, 2018), em uma ecologia propícia à exploração.

Pensando nos contextos amazônicos, isso se torna significativamente problemático, tendo em vista que, somadas às ausências de políticas sensíveis para com suas particularidades, diversas comunidades são diariamente ameaçadas (fisicamente, e psicologicamente) no que diz respeito à preservação de seus modos de vida, de suas identidades. A passagem abaixo, de Brenda Cardoso de Castro 
(2020, não publicado), exemplifica tais violações presentes no cotidiano em várias regiões da Amazônia:

Enquanto a Amazônia, no singular e no substantivo feminino, é subjugada no projeto de nação como obstáculo e ao mesmo tempo solução para o problema do Brasil, a saber, o subdesenvolvimento, as vidas e culturas das populações amazônidas continuam sendo atropeladas por políticas e megaprojetos desenvolvimentistas, que, há décadas, têm demonstrado que para se ter acesso a alguns direitos básicos é preciso abrir mão de direitos basilares como a autonomia. O saneamento, a luz, a educação e a saúde são precarizados a ponto de se ter que desejar o capitalismo para que haja uma esperança de garantia de direitos, como uma espécie de venda -casada feita pela aliança entre o Estado e o capital, ainda que a promessa pouco ou nada se cumpra em muitos casos (p. 323).

Esta aliança entre o Estado e o capital se encontra inserida em um sistema político-econômico desenhado em uma lógica "de cima para baixo", tendo em vista a forma como as motivações econômicas por (muitas) vezes se sobrepõem às demandas sociopolíticas existentes. Em seu estudo, Mills (2018) discute como Organizações Internacionais de caráter econômico, como o Banco Mundial, participam da tomada de decisões em assuntos ligados ao desenvolvimento internacional considerando engajamentos puramente econômicos.

Aplicada a agenda em saúde mental global, a economia do cuidado age no sentido de criar um "paradigma da produtividade capacitista", no qual as ações de cuidado em saúde mental devem ser feitas para que, com isso, a produtividade da mão-de-obra não seja prejudicada. É neste sistema que os Objetivos do Desenvolvimento Sustentável se encontram embasados, logo, para além de criticar a ainda incipiente abrangência da saúde mental nas ODS, não deveríamos criticar o paradigma de desenvolvimento que baseia tudo?

Assim, um desenvolvimento que paute seus esforços em satisfazer as necessidades fundamentais das populações deve ser considerado enquanto uma alternativa. Mas como realizar isso? Em primeiro lugar, como já apresentado ao longo do artigo, a saúde mental deve ser compreendida enquanto um constructo multifacetado e multidirecional, ou seja, não focado exclusivamente no indivíduo, mas em todas as relações que este partilha (com outros indivíduos, com o ambiente social, ambiente ecológico, dentre outros). Dessa forma, uma agenda pensada com base em 
intervenções que abranjam problemáticas voltadas ao desenvolvimento (em uma escala humana) e em saúde mental pode produzir respostas com maiores benefícios para as populações em distintas situações de vulnerabilidade, como apontam estudos realizados (LUND, 2018; MILLS, 2018; SAXENA et al., 2019).

Contudo, com vistas a evitar que a lógica circular de exclusão de participação e ativismo das comunidades em contextos amazônicos persista, este artigo propõe uma reflexão à luz de um paradigma para além do desenvolvimento sustentável. Afinal, enquanto um sistema de valores normativos, só é possível pensar criticamente o desenvolvimento sustentável quando identificamos as experiências antecessoras que possibilitaram a construção de tal paradigma, e vislumbramos possibilidades futuras ao mesmo.

\section{4 É possível viver para além do desenvolvimento?}

Considerando o que foi apresentado ao longo do texto, é possível que a impressão permanente seja que necessitamos urgentemente de mudanças estruturais. De fato, o modo de pensar o desenvolvimento invisibilizando e/ou excluindo grupos sociais vulneráveis deve ser repensado, mas de que modo? Como é possível transformar algo que parece, a princípio, tão acima das pessoas? Afinal, é possível viver para além da ideia de desenvolvimento? Experiências latinoamericanas mostram que sim. Ailton Krenak (2019) aponta que, em primeiro lugar, é necessário realizar um exercício de reflexão:

Como justificar que somos uma humanidade se mais de $70 \%$ estão totalmente alienados do mínimo exercício de ser? A modernização jogou essa gente do campo e da floresta para viver em favelas e em periferias, para virar mão de obra em centros urbanos. Essas pessoas foram arrancadas de seus coletivos, de seus lugares de origem, e jogadas nesse liquidificador chamado humanidade (p. 14).

A fala de Krenak, membro da etnia indígena krenak, ambientalista e escritor, aponta como a busca (diga-se desenfreada) por um estado de desenvolvimento, contribuiu para a perda do exercício de ser e estar de diversas pessoas. Max-Neef, Elizalde e Hopenhayn (2010) apresentam, ao buscarem uma Matriz de Necessidades Humanas Fundamentais, quatro categorias de necessidades, ditas existenciais: ser, 
ter, fazer e interagir. Tais categorias axiológicas (com exceção da categoria ter) parecem ser incompatíveis com o paradigma de desenvolvimento vigente em nossa sociedade ocidental contemporânea, como afirma Krenak (2019). Em uma tentativa de desvencilhamento de tal percepção, a proposta do Bien Vivir (ou Buen Viver ou ainda Bem Viver) é pensada enquanto uma alternativa que enfoca nas cosmovisões de saberes tradicionais, largamente marginalizados.

O Bien Vivir pode ser entendido enquanto um conjunto de práticas que são orientadas para a construção, reprodução e transmissão de conhecimentos, cosmovisões baseadas na convivência entre o ser humano e a natureza, na interculturalidade e o respeito às diversidades (GUDYNAS; ACOSTA, 2011; COSTA; KÜHN, 2019). A proposta do Bien Viviré apresentada como contrária à colonização do poder realizado pelo Norte Global, em relações que ultrapassam os passados coloniais e atravessam práticas exploratórias neocoloniais.

Segundo Gudynas e Acosta (2011), ainda que sejam necessários dispositivos relacionados ao Estado e ao Mercado, o Bien Vivir é uma alternativa ao modelo atual de desenvolvimento internacional, tendo em vista que nenhum dos dois é utilizado enquanto o único componente da vida social e política das pessoas. Relembrando a citação do estudo de Cardoso de Castro (2020, não publicado), acerca da "venda casada" realizada entre Estado e Capital para a satisfação (não completa) de necessidades básicas, comunidades pautadas no Bien Vivir possuem uma maior possibilidade de satisfação de suas necessidades fundamentais.

Em linhas gerais, a proposta do Bien Vivir não deve ser vista enquanto algo que venha a se opor ao ideário de desenvolvimento a qual nossas políticas se baseiam atualmente, e sim, como uma proposta reformuladora, a partir de bases a exemplo da justiça social e da coexistência entre pessoa e ambiente, bem como o respeito às cosmovisões tradicionais (VANHULST; BELING, 2014; COSTA; KÜHN, 2019). Mas como tudo isso aplicaria à saúde mental?

Como já apresentado ao longo do presente estudo, este constructo apresenta uma série de limitações, logo advogamos por uma ecologia do cuidado, ou seja, uma perspectiva que entenda o cuidado enquanto uma prática holística, sistêmica,

\footnotetext{
${ }^{4}$ Uma significativa literatura acerca do Bien Vivir vêm sendo produzida na última década, para conhecer com maior profundidade de detalhes, por favor, consulte: ESCOBAR (2009); GUDYNAS \& ACOSTA (2011), QUIJANO (2012); CUBILLO-GUEVARA, HIDALGO-CAPITÁN \& GARCÍA-ALVAREZ (2016); HIDALGO-CAPITÁN \& CUBILLO-GUEVARA (2017), dentre outros.
} 
descolonizadora do indivíduo para si próprio e para sua comunidade. Uma "revolução do bem-estar" nas palavras de Saamah Abdallah (2010). Articulados à proposta das necessidades humanas fundamentais (MAX-NEEF; ELIZALDE; HOPENHAYN, 2010), a possibilidade da construção de práticas que possibilitem a expressão de ser, ter, fazer e estar das pessoas poderia possibilitar a constituição holística proposta aqui.

\section{Considerações Finais}

Frente ao que foi apresentado enquanto discussão entre: construções sobre desenvolvimento sustentável, as realidades amazônicas e o conceito de saúde mental, podemos salientar que os objetivos do presente artigo foram contemplados, e que a relação entre desenvolvimento sustentável e saúde mental na amazônia é uma demanda emergencial a níveis regionais e globais. As produções científicas apontam a necessidade de repensar o papel da saúde mental no desenvolvimento sustentável, e as medidas relacionadas a Amazônia por sua vez, precisam ser contextualizadas a níveis sociais, políticos, territoriais, econômicos e culturais.

Enquanto corpo de pesquisadores, nossas produções científicas a respeito de saúde na Amazônia, principalmente com relação a saúde mental devem ser repensadas. Desvencilhando nossas discussões de agendas político-sociais, a efetivação pela melhoria das condições de bem-viver para os diversos grupos sociais se transforma em um conjunto de conceitos esvaziados, distantes às realidades, seja para $\circ$ que se entende enquanto desenvolvimento, seja para o que se entende enquanto sociedade.

Enquanto principais limitações, devemos salientar a ainda incipiente produção científica entre os Objetivos do Desenvolvimento Sustentável e problemáticas em contextos amazônicos. Tal fato está relacionado à percepção ainda existente de que as únicas problemáticas na região são de cunho ambiental (visto a significativa frequência de estudos que abordam em específico ODS ligadas ao meio ambiente, em um paradigma ainda limitado). Ademais, a dificuldade na busca de estudos empíricos que retratassem intersecções com o tema pesquisado também é um ponto que deve ser discutido. Por fim, a falta de dados estatísticos acerca da saúde, em especial a mental, de populações tradicionais na Amazônia é um problema histórico e recorrente. Essa dificuldade pode ser vista como uma falta de articulação entre 
serviços públicos, o Estado, as políticas públicas construídas e demais segmentos de saúde.

Considerando isso, sugerimos que investigações futuras pesquisem de que modo a busca pelo desenvolvimento sustentável é uma realidade efetiva para os contextos amazônicos. De fato, as ODS abrem caminhos às demandas reais de necessidades de comunidades na Amazônia? Na prática, como esse conjunto de valores normativos pode se alinhar às realidades de quem aqui vive? Como propostas alternativas, como o Bien Vivir, podem ser incorporadas em uma agenda de pesquisas e de políticas, para contribuir ao bem-estar da população?

Tais questionamentos, dentre outros que o presente estudo possa suscitar, devem ser investigados à luz de discussões que valorizem não apenas um ideal de identidade amazônica, compartilhada e comercializada desde os seus primeiros atos exploratórios, mas sim, reconhecendo o histórico de lutas, e para além, na desestabilização de percepções consideradas como imutáveis, nos afastando da ideia (ainda existente e persistente) de Eldorado, nos permitindo assim, dialogar com distintas formas de ser e estar no mundo.

\section{REFERÊNCIAS}

ABDALLAH, Saamah. La revolución del bienestar. In: SEMPERE, Joaquim; ACOSTA, Alberto; ABDALLAH, Saamah; ORTÍ, Mario. Enfoques sobre bienestar y buen vivir. Madrid: Centro de Investigación para la Paz (CIP-Ecosocial). 2010. 56 p.

AGUADO, Guillermo; CABEZA, Maitane; CASTILLO, Julio. Enseñanzas del Buen Vivir para construir una Pedagogía del Cuidado. REVISTA INTERNACIONAL sobre INVESTIGACIÓN en EDUCACIÓN GLOBAL y para el DESARROLLO. n. 10, p. 71 80, 2016.

ANDRADE, Antonio Luiz Menezes. Indicadores de sustentabilidade na Reserva de Desenvolvimento Sustentável do Piranha, Manacapuru, Amazonas, Brasil. Acta Amazônica, v. 37, n. 3, p. 401-412, 2007. http://dx.doi.org/10.1590/S004459672007000300011

BASTER, Raquel. O silenciamento das mulheres camponesas em situação de conflitos no campo e as sementes que anunciam suas resistências. p.83-88. In: CPT. Conflitos no campo Brasil 2018. Goiânia: Comissão Pastoral da Terra, 2019.

BATISTA, Eraldo Carlos; FERNANDES, Dayane Fernandes; BATISTA, Luana Karoline da Silva. O Cuidado em Saúde Mental na Perspectiva de Profissionais de um CAPS I na Amazônia. Pluralidades em Saúde Mental, Curitiba, v. 7, n. 1, p. 77-92, jan./jun. 2018. 
BATISTA, Marianna Queiróz. Saúde mental em contextos indígenas: Escassez de pesquisas brasileiras, invisibilidade das diferenças. Estudos de Psicologia, v. 21, n. 4, p. 403-414, 2016.

BRASIL. Plano Amazônia Sustentável: diretrizes para o desenvolvimento sustentável da Amazônia. Brasília: Ministério do Meio Ambiente, 2008. 112 p.

. Saúde Mental e Economia Solidária: inclusão social pelo trabalho. Ministério da Saúde, Secretaria de Atenção à Saúde, Departamento de Ações Programáticas e Estratégicas. Brasília: Editora do Ministério da Saúde, 2005. 134 p.

CARDOSO DE CASTRO, Brenda Thainá. Mulheres Descolonizando a Amazônia pelos Caminhos de Vida no Tapajós: produção de subjetividades atravessadas pelo projeto de nação desenvolvimentista. Tese (Doutorado em Sociologia e Antropologia). Belém: Universidade Federal do Pará, 2020. 378 p. (não publicado).

CHILTON, Mariana \& JONES, Sonya. The Rights of Nature and the Future of Public Health. American Journal of Public Health. v. 110, n..4, p. 459-460, 2020.

COOPER, Sara. Global mental health and its critics: moving beyond the impasse. CriticalPublic Health, v. 26, n. 4, pp. 355-258, 2016. doi: 10.1080/09581596.2016.1161730

COSTA, Ana Monteiro; KÜHN, Daniela Dias. Bien Vivir/Buen Viver/Bem Viver: uma proposta de pós-desenvolvimento nas Epistemologias do Sul. Revista IDeAS, v. 11, n. 1-2, p. 34-66, 2017.

CUBILlO-GUEVARA, Ana Patricia; HIDALGO-CAPITÁN, Antonio Luis; GARCíAÁLVAREZ, Santiago. El Buen Vivir como alternativa al desarrollo para América Latina. Iberoamerican Journal of Development Studies, v. 5, n. 2, p. 30-57, 2016.

CUETO, Marcos. Saúde Global: uma breve história. Rio de Janeiro: Editora Fiocruz, 2015, 120 p.

DJONÚ, Patricia; RABELO, Laudemira Silva; LIMA, Patrícia Verônica Pinheiro Sales; SOUTO, Michael Vandesteen Silva; SABADIA, José Antônio Beltrão; SUCUPIRA JÚNIOR, Paulo Ricardo Gorayeb. Objetivos do Desenvolvimento Sustentável e Condições de Saúde em Áreas de Risco. Ambiente \& Sociedade, São Paulo, v. 21, p. 1-20, 2018.

FURTADO, Celso. Análise do modelo brasileiro. Rio de Janeiro: Civilização Brasileira. 1972. 3르. ed. . Formação econômica do Brasil. São Paulo: Cia. Editora Nacional. 1982. 
GIANNOU, Dimitra. "Normalized Absence, Pathologized Presence"- Understanding the Health Inequalities of LGBT People in Greece. Tese de Doutorado. Universidade de Durham. 2017. 295 p.

GUDYNAS, Eduardo; ACOSTA, Alberto. El buen vivir o la disolución de la idea del progreso. In: ROJAS, Mariano. La Medición del Progreso y del Bienestar- Propuestas desde América Latina. Cidade do México, México: Foro Consultativo Cientifico y Tecnológico. 2011.

GUILLEN-ROYO, Mònica; GUARDIOLA, Jorge \& GARCIA-QUERO, Fernando. Sustainable development in times of economic crisis: A needs-based illustration from Granada (Spain). Journal of Cleaner Production, v. 150, 2017, p. 267-276. https://doi.org/10.1016/j.jclepro.2017.03.008

GUREJE, Oye; THORNICROFT, Graham. Health equity and mental health in post2015 sustainable development goals. TheLancetPsychiatry, v. 2, n. 1, p. 12-14, 2015. doi:10.1016/s2215-0366(14)00094-7

HIDALGO-CAPITÁN, Antonio Luis; CUBILLO-GUEVARA, Ana Patricia. Deconstrucción y genealogía del "buen vivir" latinoamericano. El (trino) "buen vivir" y sus diversos manantiales intelectuales. International Development Policy [Online], n. 9, p. 1-25. 2017. doi: 10.4000/poldev.2517

HOLDEN, Erling; LINNEURD, Kristin \& BANISTER, David. The Imperatives of Sustainable Development. Sust. Dev., n. 25, 2017, p. 213-226. doi: 10.1002/sd.1647

HUBER, Matcheld. Towards a new, dynamic concept of health-lts operationalisation and use in public health and healthcare, and in evaluating health effects of food. 2014. Disponível em: http://www.nvag.nl/afbeeldingen/2014/Thesis\%20Machteld\%20Huber.pdf. Acesso em 15 maio 2020.

INSTITUTO DE PESQUISA ECONÔMICA APLICADA. ODS 3- O que mostra o retrato do Brasil?. Brasília, DF: Livraria IPEA. 2019. 46 p.

IZUTSU, Takashi; TSUTSUMI, Atsuro; MINAS, Harry, THORNICROFT, Grahan, PATEL, Vikram; ITO, Akiko. Mental health and wellbeing in the Sustainable Development Goals. The Lancet Psychiatry, v. 2, n. 12, p. 1052-1054, 2015. doi:10.1016/s2215-0366(15)00457-5

KRENAK, Ailton. Ideias para adiar o fim do mundo. São Paulo: Companhia das Letras, 2019.

LOUREIRO, Violeta Refkalefsky. Amazônia: uma história de perdas e danos, um futuro a (re)construir. Estudos Avançados, v. 16, n. 45, p. 107-121, 2002. 
LUND, Crick; BROOKE-SUMNER, Carrie; BAINGANA, Florence; BARON, Emily Claire; BREUER, Erica; CHANDRA, Prabha; HAUSHOFER, Johannes; HERRMAN, Helen; JORDANS, Mark; KIELING, Christian; MEDINA-MORA, Maria Elena; MORGAN, Ellen; OMIGBODUN, Olayinka; TOL, Wietse; PATEL, Vikram; SAXENA, Shekhar. Social determinants of mental disorders and the Sustainable Development Goals: a systematic review of reviews. The Lancet Psychiatry, v. 5, n. 4, p. 357-369, 2018. doi:10.1016/s2215-0366(18)30060-9

MADEIRA, Welbson do Vale. Plano Amazônia Sustentável e Desenvolvimento Desigual. Ambiente \& Sociedade n São Paulo v. XVII, n. 3, p. 19-34. 2014.

MATTA, Gustavo Corrêa. A Organização Mundial da Saúde: do controle de epidemias à luta pela hegemonia. Trabalho, Educação e Saúde. v. 3, n. 2, p. 371-396, 2005.

MASTEL, Molly; BUSSALLEU, Alejandra; PAZ-SOLDÁN, Valerie A.; SALMÓNMULANOVICH, Gabriela; VALDÉS-VELÁSQUEZ, Armando \& HARTINGER, Stella M. Critical linkages between land use change and human health in the Amazon region: $A$ $\begin{array}{lllll}\text { scoping } & \text { review. PLoS ONE 13(6): }\end{array}$ https://doi.org/10.1371/journal.pone.0196414

MAX-NEEF, Manfred; ELIZALDE, Antonio; HOPENHAYN, Martín. Desarrollo a Escala Humana: Opciones para el Futuro. Madrid: Biblioteca CF+S, 2010.

MCINNES, Colin; LEE, Kelley. Global Health \& International Relations. Cambridge: Polity Press. 2012. 219 p.

MEDEIROS, Marcílio Sandro de; AUGUSTO, Lia Giraldo da Silva; BARCA, Stefani; SACRAMENTO, Daniel Souza; SANTIAGO NETA, Inez Siqueira; GONÇALVES, Isabela Cristina; COSTA, André Monteiro. A saúde no contexto de uma reserva de desenvolvimento sustentável: o caso Mamirauá, na Amazônia brasileira. Saúde \& Sociedade, 27(1), 128-148, 2018. https://doi.org/10.1590/s0104-12902018170514

MILLS, China. From 'Invisible Problem' to Global Priority: The Inclusion of Mental Health in the Sustainable Development Goals. DevelopmentandChange 49(3): 843866. DOI: 10.1111/dech.12397. 2018

MORENO, Nicole Silva; SILVA, Denys Daniel da; SOUZA, Washington José de. Desenvolvimento à Escala Humana na economia solidária: síntese de necessidades e satisfatores de trabalhadoras do segmento da produção de artesanato (Natal, 2017). In: VI Conferência Internacional de Pesquisas e Estudos sobre Economia Social e Solidária, 2017, Manaus. CIRIEC 2017, 2017.

MORETTI-PIRES, Rodrigo Otávio; CORRADI-WEBSTER, Clarissa Mendoça; FURTADO, Erikson Felipe. Consumo de álcool e atenção primária no interior da Amazônia: sobre a formação de médicos e enfermeiros para assistência integral. Revista Brasileira de Educação Médica, v. 35, n. 2, p. 219-228, 2011. https://doi.org/10.5964/ijpr.v9i2.187 
NORDENFELT, Lennart. Understanding the Concept of Health. 2007. Disponível em: http://www.fil.lu.se/hommageawlodek/site/papper/NordenfeltLennart.pdf. Acesso em 18 maio 2020.

ORGANIZAÇÃO DAS NAÇÕES UNIDAS. Agenda 2030- Desenvolvimento Sustentável. 2019. Disponível em: https://nacoesunidas.org/pos2015/agenda2030/. Acesso em 20 maio 2020.

ORGANIZAÇÃO MUNDIAL DA SAÚDE. Livro de Recursos da OMS sobre Saúde Mental, Direitos Humanos e Legislação: cuidar, sim - excluir, não. Genebra, Suíça: OMS, $2005.229 \quad$ p. 20 Disponível em: http://www.who.int/mental_health/policy/Livroderecursosrevisao_FINAL.pdf. Acesso em 25 maio 2020.

PATEL, Vikram; SAXENA, Shekhar; FRANKISH, Helen; BOYCE, Niall. Sustainable development and global mental health- a Lancet Commission. The Lancet Psychiatry, v. 387, p. 1143-1145, 2016.

PROGRAMA DAS NAÇÕES UNIDAS PARA O DESENVOLVIMENTO. Objetivos do Desenvolvimento Sustentável. $2019 . \quad$ Disponível em: https://www.br.undp.org/content/brazil/pt/home/sustainable-developmentgoals.html.Acesso em 18 maio 2020.

PELENC, Jérôme; DUBOIS, Jean-Luc. Human Development and Strong Sustainability: A Mutual Dialogue. In: CRABTREE, Andrew. (Ed.). Sustainability, Capabilities and Human Security. Palgrave Macmillan. 2020. 184 p. doi:10.1007/9783-030-38905-5

REGAZZONI, Carlos Javier. La política de la salud global: agenda, actores y perspectivas. Buenos Aires: Consejo Argentino para las Relaciones Internacionales. 2007.

RIBEIRO, Gustavo Lins. Ambientalismo e Desenvolvimento Sustentado. Nova Ideologia/Utopia Do Desenvolvimento. Revista De Antropologia, São Paulo, USP, n. 34, p. 59-101. 1981. https://doi.org/10.11606/2179-0892.ra.1991.111253

ROCHA, Evangelista. Saúde e Objetivos do Desenvolvimento Sustentável. Revista Factores de Risco, n. 45, p. 12-19, 2017.

RUEDIGER, Marco Aurélio; JANNUZZI, Paulo de Martino (coords.). Políticas públicas para o desenvolvimento sustentável: dos mínimos sociais dos objetivos de desenvolvimento do milênio à agenda multissetorial e integrada de desenvolvimento sustentável. Rio de Janeiro : FGV DAPP, 2018. 1 recurso online (82 p.) : PDF, il.

SANTOS, Edinilza Ribeiro dos; HUANG, Hsiang; MENEZES, Paulo Rossi \& SCAZUFCA, Marcia. Prevalence of Depression and Depression Care for Populations Registered in Primary Care in Two Remote Cities in the Brazilian Amazon. PLoS ONE 11(3): e0150046. doi:10.1371/journal.pone.0150046 
SCHWEICKARDT, Júlio Cesar; FERLA, Alcindo Antonio; CECCIM, Ricardo Burg; LIMA, Rodrigo Tobias de Sousa; KADRI, Michele Rocha; OLIVEIRA, Paulo de Tarso Ribeiro de. A Amazônia como Lugar de Produção de Redes de Cuidado. In: SCHWEICKARDT, Júlio Cesar; FERLA, Alcindo Antonio; CECCIM, Ricardo Burg; LIMA, Rodrigo Tobias de Sousa; KADRI, Michele Rocha; OLIVEIRA, Paulo de Tarso Ribeiro de (orgs.). Educação e práticas de saúde na Amazônia: tecendo redes de cuidado. Porto Alegre: Rede Unida, 2016. 316 p.

SAXENA, Shekhar; KESTEL,Devora; SUNKEL, Charlene; LONDON, Elisha; HORTON, Richard; PATEL, Vikram; SWAMINATHAN, Soumya. Countdown Global Mental Health 2030, The Lancet Psychiatry, v. 393, 2019. http://dx.doi.org/10.1016/S0140-6736(19)30424-6

SILVEIRA, Matheus dos Santos da. A Construção de Práticas Discursivas de Atenção à Saúde Mental da Organização Mundial da Saúde referente à comunidade LGBTQI. Trabalho de Conclusão de Curso (Bacharelado em Relações Internacionais). Belém: Universidade da Amazônia. 2018. 118 p.

SILVEIRA, Rodrigo Pinheiro; PINHEIRO, Roseni. Entendendo as Necessidades de Médicos no Interior da Amazônia- Brasil. Revista Brasileira de Educação Médica, v. 38, n. 4, p. 451-459. 2014.

TALLMAN, Paula Syke. Water insecurity and mental health in the Amazon: Economic and ecological drivers of distress. Economic Anthropology, "Water and Economy" 2019; 6: 304-316 DOI:10.1002/sea2.12144

THORNICROFT, Graham; PATEL, Vikram. Including mental health among the new sustainable development goals. BMJ, n. 349, 2014. doi:10.1136/bmj.g5189

VAN BELLEN, Hans Michael. (2004). Desenvolvimento Sustentável: Uma Descrição das Principais Ferramentas de Avaliação. Ambiente\&Sociedade, v.7(1), 67-87.

VANHULST, Julien; BELING, Adrian E. Buenvivir: Emergent discourse within or beyond sustainable development? Ecological Economics, n. 101, p. 54-63. 2014. doi:10.1016/j.ecolecon.2014.02.017

VÉLEZ, Eliana María Montoya; LÓPEZ RIOS, Jennifer Marcela; MARULANDA, Sergio Cristancho; VALENCIA FRANCO, Marcela Cristina; DE LA ROSA, Oscar David Montero \& HOLGUín, Dora María Hernández. Aproximación a la concepción de la salud mental para los pueblos indígenas de Colombia. Ciência \& Saúde Coletiva, 25(3): 1157-1166, 2020. DOI: 10.1590/1413-81232020253.17832018

VIEIRA, Ima Célia Guimarães. Abordagens e desafios no uso de indicadores de sustentabilidade no contexto amazônico. Cienc. Cult., São Paulo , v. 71, n. 1, p. 4650, Jan. 2019 . http://dx.doi.org/10.21800/2317-66602019000100013.

VIEIRA, Janete Maria Rebelo; GARNELO, Luíza; HORTALE, Virginia Alonso. A. Análise da atenção básica em cinco municípios da Amazônia ocidental, com ênfase 
no Programa Saúde da Família. Revista Saúde \& Sociedade, v. 19, n. 4, p. 852-865, 2016. http://dx.doi.org/10.1590/S0104-12902010000400012

VITA, Gibran; HERTWICH, Edgar G.; STADLER, Konstantin \& WOOD, Richard. Connecting global emissions to fundamental human needs and their satisfaction. Environ. Res. Lett., n. 14, 2019, p. 1-17. https://doi.org/10.1088/1748-9326/aae6e0

VOTRUBA, Nicole; THORNICROFT, Graham. Sustainable development goals and mental health: learnings from the contribution of the FundaMentalSDG global initiative. Global Mental Health, v. 3, n. e26, p. 1-6, 2016. doi:10.1017/gmh.2016.20

VOTRUBA, Nicole; EATON, Julian; PRINCE, Martin; THORNICROFT, Graham. The importance of global mental health for the Sustainable Development Goals, Journal of Mental Health, v. 23, n. 6, p. 283-286, 2014. doi: 10.3109/09638237.2014.976857

WUTICH, Amber; BREWIS, Alexandra. Food, Water, and Scarcity: Toward a Broader Anthropology of Resource Insecurity. CurrentAnthropology, v. 55, n. 4, p. 444-468, 2014. 